\title{
MEJORA EN EL APROVECHAMIENTO DEL ESPACIO CÚBICO DE CONTENEDORES DE EXPORTACIÓN DE MANGUERAS EN UNA EMPRESA AGRO INDUSTRIAL EN EL PERÚ
}

\section{Enhancing use of the cubic space of hose export containers in an agro-industrial company in Perú}

\author{
Cristhian Giancarlo Aradiel Abad¹, Diego Ángel Dávila Vilchez², Tania Sthefany Gamboa Rojas³, \\ Gabriela Paola Veliz Ponce ${ }^{4}$ \\ 1-4Pontificia Universidad Católica Del Perú, Escuela de Ingeniería Industrial, Grupo de Investigación \\ IISE PUCP, Perú.Email: ${ }^{1}$ cristhian.aradiel@pucp.pe, ${ }^{2}$ diego.davila@pucp.edu.pe, \\ 3tania.gamboa@pucp.edu.pe, ${ }^{4}$ gabriela.veliz@pucp.edu.pe
}

(Recibido febrero 06 de 2021 y Aceptado junio 15 de 2021)

\begin{abstract}
Resumen
El presente artículo describe la situación actual de la exportación de mangueras hidráulicas en el Perú, analiza los principales factores que incrementan el costo de exportación y propone un modelo de programación lineal para determinar el tipo de container que debe ser usado según variables expuestas.
\end{abstract}

Palabras clave: optimización, programación lineal, espacio cúbico.

\begin{abstract}
This article describes the current situation of the export of hydraulic hoses in Peru, analyzes the main factors that increase the export cost and proposes a linear programming model to determine the type of container to use according to the exposed variables.
\end{abstract}

Key words: optimization, linear programming, cubic space.

\section{INTRODUCCIÓN}

En la actualidad, existen diversas empresas exportadoras que planifican la consolidación de sus despachos y la distribución de sus productos, de manera empírica desde su planta principal hacia distintos puntos de venta a nivel internacional. El caso de estudio de la presente investigación es de una empresa exportadora peruana, que cuenta con diversos productos (línea de goteros, aspersores, emisores, tuberías, conectores y accesorios) para el riego de cultivos. El flujo de contenedores es la principal motivación que moviliza la cadena de suministro, de tal manera que, se pueda satisfacer la exigente demanda de los mercados de diferentes regiones, desde el requerimiento de una logística internacional relevante.
En ese sentido, la principal desventaja de la consolidación de los productos es la necesidad de movilizarlos en paletas y contenedores, proceso que se gestiona acorde a la experiencia del operador logístico de la empresa. Esta práctica incurre en altos costos logísticos puesto que no se aprovecha la capacidad total de los contenedores y no existe un estándar de paletizado. Por esta razón, no se usa de manera óptima el espacio ni la asignación de la carga de acuerdo con el tipo de contenedor 40 ST (FCL o LCL). La estrategia inicial es optimizar el paletizado y consolidación de la carga, es así que se propone la creación de un modelo matemático a través de la programación lineal, que optimice la consolidación de los productos en los contenedores y reduzca los costos de operaciones logísticas del envío marítimo hasta su destino final. 


\section{ANTECEDENTES}

En Perú, el sector agroexportador refleja una participación de $14.3 \%$ en promedio con US $\$ 10,000$ millones para el 2021, el cual ilustra una expectativa suficiente de oportunidad para invertir (Ministerio de Agricultura y Riego, 2020) [2]. El presente caso de estudio es sobre una empresa agroexportadora peruana, la cual posee cinco grupos de negocios que ofrecen soluciones innovadoras en diversas industrias. Estas incluyen sectores como construcción e infraestructura, comunicaciones de datos, productos químicos y más. La cual ocupa el primer lugar en ventas de la industria de riego por goteo y tiene como principales competidores a Naan Dan Jain y Jhon Deer Water.

La principal actividad de la empresa se centra en ofrecer productos y soluciones de riego tecnificado; monitoreo, control digital de campo abierto, huertos y cultivos protegidos. Cabe resaltar que está enfocada en abordar uno de los mayores desafíos del mundo: escasez de agua y alimentos. Otras actividades en las que incursiona son el soporte técnico y agronómico para proyectos de cliente, además de impulsar iniciativas para mejorar la calidad de cultivos y el incremento del rendimiento y uso eficiente del agua. [3]

\section{METOdOLOGÍA}

La metodología propuesta se detalla en las siguientes actividades:

\subsection{Mapeo de procesos}

Esta actividad permitió conocer el proceso de exportación de mangueras, accesorios, aspersores, etc. involucrando diversas áreas desde la solicitud de la orden y servicio al cliente, pasando por áreas de producción, planeamiento, comercio exterior almacenes y distribución hasta el despacho y envío de la documentación al cliente. Además, el flujo de las mangueras de exportación proviene de una planificación pronosticada del abastecimiento de los países destino con la finalidad de minimizar el impacto de las roturas de stock. Cabe resaltar que la presente investigación y mejora se enfocará en las actividades del operador logístico (in house) relacionadas a la consolidación de carga paletizada en containers.

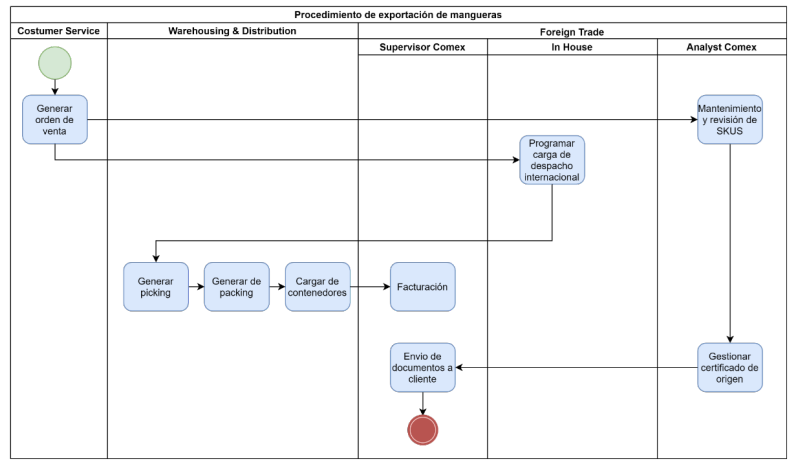

Figura 1. Hose Export Process

\subsection{Recolección y análisis de información}

Se recolectó información relacionada con los pesos $(\mathrm{kg})$, volumen (m3) y venta de los SKUS en los distintos canales a través de la demanda. Para ello se identificó la estructura de la red logística con respecto a los tiempos relacionados al envío de los productos por destino y por SKU por cuestiones prácticas de la empresa agroindustrial. En resumen, se consolidó la información y un análisis de la demanda para determinar en qué SKU's generar mayor valor a la compañía. En ese sentido, se estableció que, de 38 productos, solo 20 tipos de mangueras generan mayor ganancia de acuerdo con el Pareto mostrado.

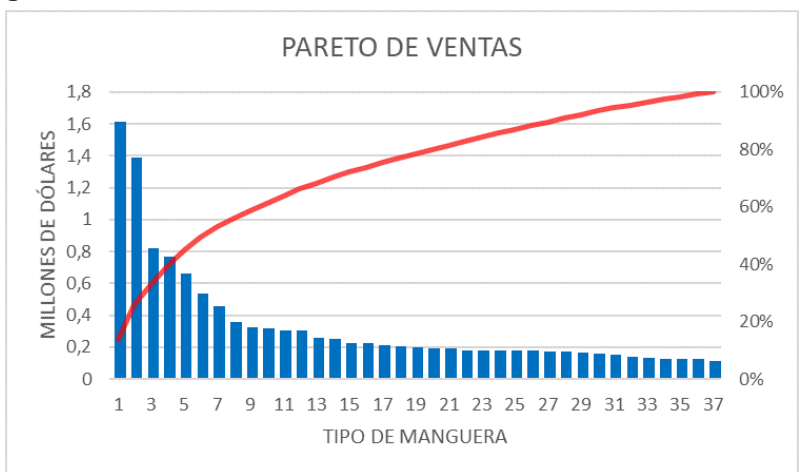

Figura 2. Pareto of hose sales.

\subsection{Construcción del modelo de optimización}

Se debe tener en cuenta que para la determinación del conjunto de SKU's y paletas, estas son asignadas al tipo 
de contenedor, no obstante, cabe resaltar que el objetivo de este modelo es el aprovechamiento del espaciamiento cúbico de los contenedores a través de carga paletizada, reduciendo así los costos del flujo de contenedores que serán exportados. La principal variable de decisión es la cantidad pronosticada de SKU's propuestos para exportación a destinos como Chile, Ecuador, Colombia, Argentina, México, Centro América y Brasil; por otro lado, las restricciones están vinculadas a la demanda de los clientes, modalidad de transporte y costos de depósito temporal, flete local, asociados al contenedor, y uso de patio en puerto, etc. (costos de logística internacional).

\subsection{Análisis de resultados y de escenarios}

Se presentarán los resultados del escenario actual, es decir de la situación actual empírica en la que el operador logístico consolida la carga y se comparara los resultados del modelo matemático propuesto.

A continuación, se presenta la estructura de costos relacionados a la exportación de contenedores (logística internacional en la cadena de suministro). La Figura 3 , representa los costos fijos en la exportación de la mercadería e independiente del flujo de contenedores:

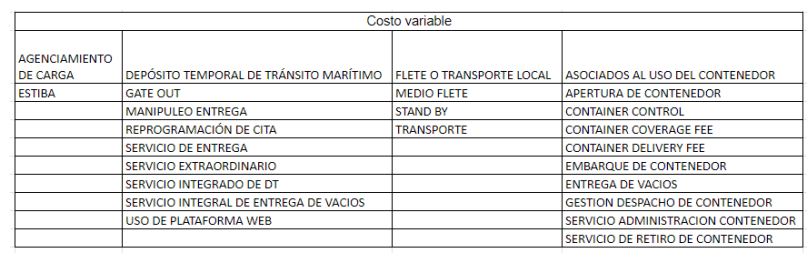

Figura 3. Costos variables asociados a la exportación de mangueras.

\begin{tabular}{|l|l|l|}
\hline \multicolumn{2}{|c|}{ COSTO FIJO } \\
\hline $\begin{array}{l}\text { AGENCIAMIENTO DE } \\
\text { ADUANA }\end{array}$ & AGENCIAMIENTO PORTUARIO MARÍTIMO & $\begin{array}{l}\text { TRÁMITES DE DOCUMENTOS Y } \\
\text { ADMINISTRATIVOS }\end{array}$ \\
\hline COMISION AGENCIA & AUXILO MECÁNICO A CAMIONES EXTERNOS INOPERATIVOS & GASTO ADMINISTRATIVO \\
\hline GASTOS DE DESPACHO & CARGO POR CITA NO UTILIZADA & GASTOS ADMINISTRATIVOS \\
\hline PRECINTO & CONTENEDOR ALTO CUBICAJE 4OHQ & TRAMITE DOCUMENTARIO EXPO \\
\hline & ENTREGA TARDIA DE DOCUMENTOS & \\
\hline & MANIPULEO GRUA & \\
\hline & MOVILIIIACION PARA BOE & \\
\hline & MOVIIIIENOS EXTRAS EN EL PATIO & \\
\hline & SCAC EXPO & \\
\hline & SERVICIO ESPECIAL DEPOSITO TEMPORAL & \\
\hline
\end{tabular}

Figura 4. Costos fijos asociados a la exportación de mangueras.
Además, se tomará en cuenta el volumen de cada producto con el objetivo de aprovechar el espaciamiento cúbico de los contenedores:

\begin{tabular}{|l|r|}
\hline & Volumen (m3) \\
\hline Manguera tipo 1 & 0,068 \\
\hline Manguera tipo 2 & 0,064 \\
\hline Manguera tipo 3 & 0,067 \\
\hline Manguera tipo 4 & 0,073 \\
\hline Manguera tipo 5 & 0,064 \\
\hline Manguera tipo 6 & 0,066 \\
\hline Manguera tipo 7 & 0,073 \\
\hline Manguera tipo 8 & 0,064 \\
\hline Manguera tipo 9 & 0,071 \\
\hline Manguera tipo 10 & 0,070 \\
\hline Manguera tipo 11 & 0,063 \\
\hline Manguera tipo 12 & 0,070 \\
\hline Manguera tipo 13 & 0,037 \\
\hline Manguera tipo 14 & 0,065 \\
\hline Manguera tipo 15 & 0,070 \\
\hline Manguera tipo 16 & 0,065 \\
\hline Manguera tipo 17 & 0,075 \\
\hline Manguera tipo 18 & 0,066 \\
\hline Manguera tipo 19 & 0,057 \\
\hline Manguera tipo 20 & 0,068 \\
\hline
\end{tabular}

Figura 5. Volumen según tipo de mangueras.

De acuerdo con la demanda histórica de los 20 tipos de manguera, se pronosticó de acuerdo con el método de promedio móvil con tendencia móvil. De acuerdo con lo anterior, se presenta el cuadro pronóstico para la demanda mensual de los 7 países que deben ser cubiertos.

Enseguida, se explicará los sets, parámetros, variables de decisión, función objetivo, restricciones para determinar los costos totales mínimos de las exportaciones de los contenedores a distribuir al mercado internacional.

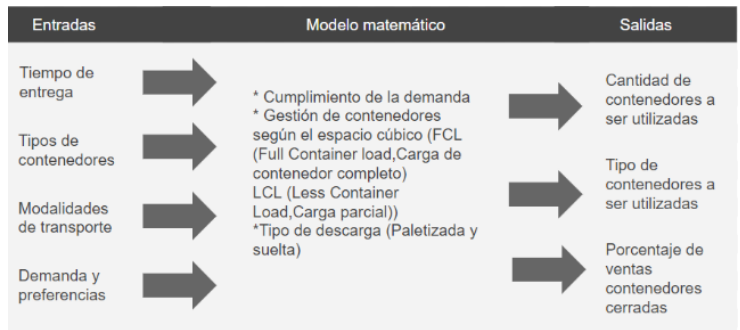

Figura 6. Variables de entrada y salida para el modelo matemático. 
El modelo de optimización se presenta en las siguientes líneas:

\section{Sets}

$$
\begin{array}{ll}
I & \text { Set of SKUs } \\
J & \text { Set of countries } \\
K & \text { Set of container types }
\end{array}
$$

\section{Parámetros}

$$
\begin{array}{ll}
d_{i} & \text { Demand SKU i } \\
v_{i} & \text { Volume SKU i } \\
{ }_{p} L C L & \text { Maximum volume allowed for an LCL container } \\
{ }_{p} F C L_{\min } & \text { Minimum volume allowed for a FCL container } \\
{ }_{p} F C L_{\max } & \text { Maximum volume allowed for a FCL container } \\
c a g & \text { Cargo agency cost } \\
c f & \text { Freight cost } \\
c d t & \text { Temporary deposit cost } \\
c a c & \text { Costs associated with the use of containers } \\
c a a & \text { Customs clearance costs and commissions } \\
c a p & \text { Costs and commissions of the port of shipment } \\
c t a & \text { Administrative costs }
\end{array}
$$

Variables

$$
\begin{array}{ll}
X_{i j k} & \begin{array}{l}
\text { Quantity of SKU i exported to country j in a } \\
\text { container type } k
\end{array} \\
W_{j k} & \begin{array}{l}
\text { Quantity of type } \mathrm{k} \text { containers exporting to } \\
\text { country j }
\end{array}
\end{array}
$$

Descripción:

$$
\operatorname{Min}(c a a+c a p+c t a)+\sum_{j \in J, k \in K}\left(W_{j k} *(c a c+c f+c d t+c a c)\right.
$$

La función objetivo busca minimizar el costo total de exportación de containers a ser distribuidos en el Mercado internacional.

Demanda de Producción

$$
\sum_{k \in K} X_{i j k}=d_{i} \quad \forall i \in I, j \in J
$$

La cantidad de SKUs a ser exportados a cada país en su respectivo tipo de container, debe ser igual a la demanda de producción mensual de SKU en general.

Límite máximo de capacidad de containers $L C L(k=1)$

$$
\sum_{i \in I} X_{i j 1} * v_{i} \leq p L C L * W_{j 1} \quad \forall j \in J
$$

El volumen total de productos que se enviarán en contenedores Less Container Load (LCL) debe ser menor o igual a la política de volumen máximo de los contenedores LCL utilizados.

Límite mínimo de capacidad de containers $F C L(k=2)$

$$
\sum_{i \in I} X_{i j 2} * v_{i} \leq p F C L_{\min } * W_{j 2} \quad \forall j \in J
$$

El volumen total de productos que se enviarán en contenedores Full Container Load (FCL) debe ser mayor o igual a la política de volumen mínimo de los contenedores FCL utilizados.

Límite máximo de capacidad de containers $F C L(k=2)$

$$
\sum_{i \in I} X_{i j 2} * v_{i} \leq p F C L_{\max } * W_{j 2} \quad \forall j \in J
$$

El volumen total de productos que se enviarán en contenedores Full Container Load (FCL) debe ser menor o igual a la política de volumen máximo de los contenedores FCL utilizados.

\section{RESULTADOS}

La minimización de contenedores que son consolidados para exportar pedidos de clientes (países), de acuerdo con la demanda de los 7 países y el desarrollo del modelo, deben realizarse de la siguiente manera: Todo el pedido desde Chile debe ser exportado en 30 contenedores Full Container Load, todo el pedido desde Ecuador en 2 Full Contenedores de carga; todo el pedido desde Colombia, 
en 5 contenedores Carga completa de contenedores; todo el pedido desde Argentina en 2 contenedores, Carga completa de contenedores; todo el pedido de México, en 1 contenedor Less Container Load; todo el pedido de Centroamérica en 30 contenedores Full Container Load; todo el pedido de Brasil, en 2 contenedores Full Container Load.

La reducción de costos de oferta internacional de productos se origina de acuerdo a la demanda del mercado latinoamericano (\$). En concordancia, los principales costos logísticos que se reducirían son los de agencia de carga, flete, almacenamiento temporal y los asociados al uso de contenedores. En el caso de la empresa exportadora, desee implementar este modelo de programación lineal, sus costos logísticos totales serían de 231.273,21 dólares, muy inferiores a sus costos actuales, que ascienden a 337.921,11 dólares.

derecha:

$$
\begin{gathered}
X_{1-2}=\frac{b \pm \sqrt{b^{2}-4 a}}{2 a} \\
x \leq \sum_{i=t}^{T} D_{i}
\end{gathered}
$$

(S\&OP), ya que la información compartida entre las áreas involucradas considera las características de los mercados internacionales.

\section{REFERENCIAS}

[1] Instituto Nacional de Estadística e Informática (2021). Tabla de actividades económicas de Clasificación Industrial Internacional Uniforme (CIIU). Revisado el 25 de abril de 2021. Disponible en: https://cdn.www.gob.pe/uploads/document/ file/1348266/Tabla_Oficial_CIIU_Rev4.pdf

[2] Comexperú (2019). Principales Preocupaciones de los Agropecuarios. Revisado el 25 de abril de 2021. Disponible en:https://www.comexperu.org pe/articulo/principales-preocupaciones-de-losagroexportadores

[3] Agroexportadora (2021). Agroexportadora, el riego de precisión cambia el panorama económico de la agricultura. Revisado el 25 de abril de 2021. Disponible en: https://www.agroexportadora.com/ es-pe/

\section{CONCLUSIONES}

El criterio empírico del operador logístico no implica necesariamente la consolidación eficiente de la carga paletizada, por lo que el uso de contenedores se puede reducir en un 31,56\%. Adicionalmente, se incrementó el cambio de contenedores $L C L$ a FCL ya que las cantidades y dimensiones de cada tipo de manguera fueron fundamentales para la mejora en el espaciamiento cúbico del 70,26\%. Esta investigación y mejora determinan una relevante disminución de costos como punto de partida y planificación para la exportación y negociación de contenedores. El modelo matemático sugiere la integración de los pronósticos de ventas de acuerdo con la metodología de Operaciones de Planificación y Ventas 